\title{
Compostos bioativos em alimentos, estresse oxidativo e inflamação: uma visão molecular da nutrição
}

\author{
Elaine dos R. Soares, ${ }^{1}$ Elisa B. Monteiro, ${ }^{1}$ Renata C. da Silva, ${ }^{1}$ Aline Batista, ${ }^{1}$ Flavia Sobreira, ${ }^{1}$ Thaísa Mattos, ${ }^{1}$ \\ Cristiane A. da Costa, ${ }^{2}$ Julio B. Daleprane ${ }^{1 *}$
}

\begin{abstract}
Resumo
A indicação de uma alimentação diversificada em frutas, legumes e verduras nos dias atuais vai além dos micronutrientes essenciais como as vitaminas e minerais que são encontrados nestes alimentos. Os compostos bioativos presentes nestes ganham cada vez mais destaque na literatura, por meio de seus promissores efeitos benéficos à saúde. Junto a isso a crescente vertente de atuação destes compostos na regulação da expressão gênica exercendo, portanto, influência no genoma humano, torna-se fundamental para o desenvolvimento de novas terapias nutricionais. Além disso, a elucidação dos mecanismos intracelulares pelos quais estes compostos exercem seus efeitos terapêuticos ratifica a compreensão dos benefícios conferidos pelos alimentos, seja na forma preventiva ou terapêutica, na tentativa de reestabelecer o desequilíbrio redox existente em um organismo. Nota-se, então, a importância das vias de sinalização molecular tais como a via do Nrf2-Keap1, uma das principais contra agentes oxidantes, e a via do NFkB, responsável pelo desencadeamento da resposta inflamatória. Desta forma, a aquisição acerca deste conhecimento se faz de suma importância haja vista que este torna possível a geração de novas fontes preventivas e terapêuticas, principalmente as envolvidas com estresse oxidativo, como as cardiovasculares, o diabetes, a hipertensão, o câncer, entre outras.
\end{abstract}

Descritores: Nutrigenômica; Compostos bioativos; Estresse oxidativo; Vias de sinalização.

\begin{abstract}
Food bioactive compounds, oxidative stress and inflammation: a molecular view of nutrition

Nowadays the indication of a diet diversification including fruits and vegetables goes beyond the essential micronutrients such as vitamins and minerals found in these foods. The bioactive compounds present in these are increasingly highlighted in the literature, through its promising beneficial health effects. Moreover the increasing strand of action of these compounds in the regulation of gene expression exercising, therefore, influence in the human genome, is fundamental to the development of new nutritional therapies. In addition, the elucidation of intracellular mechanisms by which these compounds exert their therapeutic effects confirms the understanding of the benefits conferred by the food, as a preventive or therapeutic way in attempt to restore the redox imbalance existing in an organism. Note then the importance of the molecular signaling pathways such as the Nrf2-Keap 1, a major pathway against oxidizing agents and
\end{abstract}

1. Departamento de Nutrição Básica e Experimental. Instituto de Nutrição. Universidade do Estado do Rio de Janeiro. Rio de Janeiro, RJ, Brasil.

2. Departamento de Farmacologia e Psicobiologia. Instituto de Biologia. Universidade do Estado do Rio de Janeiro. Rio de Janeiro, RJ, Brasil.

*Endereço para correspondência: Instituto de Nutrição, UERJ.

Rua São Francisco Xavier, 524, 12ㅇandar

Rio de Janeiro, RJ, Brasil. CEP: 20550-900.

E-mail: juliobd@gmail.com

Revista HUPE, Rio de Janeiro, 2015;14(3):64-72

doi: 10.12957/rhupe.2015.19942

Recebido em 19/03/2015. Aprovado em 17/07/2015.

NFkB pathway, responsible for triggering the inflammatory response. Thus, the acquisition about this knowledge is of paramount importance given that this makes it possible to generate new preventive and therapeutic sources, mainly involved with oxidative stress, such as cardiovascular, diabetes, hypertension, cancer, among others.

Keywords: nutrigenomics, bioactives compounds, oxidative stress, signal transduction..

\section{Resumen}

Compuestos bioactivos de los alimentos, el estrés oxidativo y la inflamación: una visión molecular de la nutrición

La indicación de una dieta diversificada de frutas y verduras de hoy va más adelante de los micronutrientes esenciales, como vitaminas y minerales que se encuentran en estos alimentos. Los compuestos bioactivos presentes en estos se destacan cada vez más en la literatura, a través de sus prometedores efectos beneficiosos para la salud. Es necesario añadir que, la creciente línea de acción de estos compuestos en la regulación de la expresión génica influyendo, por lo tanto, en el genoma humano, es fundamental para el desarrollo de nuevas terapias nutricionales. Además, la elucidación de los mecanismos intracelulares por los cuales estos compuestos ejercen sus efectos terapéuticos confirma la comprensión de los beneficios brindados por los alimentos, sea en su forma preventiva o terapéutica, en un intento de restablecer el desequilibrio redox existente en un organismo. Entonces es nota- 
ble la importancia de las vías de señalización molecular, tales como la via de Nrf2-Keap1, una de las principales frente a los agentes oxidantes, y la de NFkB, responsable por desencadenar la respuesta inflamatoria. Por lo tanto, la adquisición de este conocimiento es de suma importancia puesto que hace que

\section{Introdução}

A produção de radicais livres constitui um processo contínuo e fisiológico, cumprindo funções biológicas relevantes. Sua produção é regulada por diferentes processos e vias metabólicas, atuando como mediadores na sinalização extra e intracelular. Porém, a produção excessiva pode conduzir a danos oxidativos. O estresse oxidativo é caracterizado pelo acúmulo intracelular de compostos reativos ao oxigênio, e ao nitrogênio, chamadas espécies reativas de oxigênio(EROs) de nitrogênio (ERN), e normalmente ocorre nas células como consequência de um desequilíbrio do sistema redox, devido ao excesso do acúmulo de espécies reativas, principalmente EROs, e pela depleção de antioxidantes (AOX) ou ambos. ${ }^{1,2}$

Uma variedade de moléculas que atuam como radicais livres (espécies químicas com um elétron desemparelhado) derivadas do metabolismo do oxigênio têm sido descritas. Normalmente, possuem meia-vida extremamente curta, são altamente reativas, produzidas em todos os sistemas biológicos e reagem facilmente com moléculas que se localizam em torno do seu sítio de formação. As EROs incluem radicais livres como o ânion superóxido $\left(\bullet \mathrm{O}_{2}-\right)$, o radical hidroxil $(\bullet \mathrm{OH})$ e radicais lipídicos, bem como espécies oxidantes não radicalares, como o peróxido de hidrogênio $\left(\mathrm{H}_{2} \mathrm{O}_{2}\right)$ e o oxigênio singlete.,

A produção de EROs pode ser realizada por diferentes vias, entretanto, a cadeia respiratória mitocondrial é a principal fonte por possuir vários centros redox, capazes de transferir um elétron da molécula de oxigênio para formar ânion superóxido. ${ }^{5,6} \mathrm{~A}$ mitocôndria também possui numerosas proteínas antioxidantes e desacopladoras (UCPs), bem como canais iônicos que modificam o gradiente de prótons. Todos esses fatores se combinam para proporcionar um controle bem ajustado do estado redox celular. Outras enzimas, como as oxidases de membrana, são também importantes fontes de EROs e espécies reativas de nitrogênio (ERN) que podem se combinar com outras moléculas e modificar a função mitocondrial. Dentre elas, destaca-se o complexo enzimático de membrana NADPH oxidase. Suas subunidades se unem sob ativação e formam uma sea posible generar nuevas fuentes preventivas y terapéuticas, principalmente involucrados con el estrés oxidante, como las cardiovasculares, diabetes, hipertensión, cáncer, entre otras.

Palabras clave: Nutrigenómica; Compuestos bioactivos; Estrés oxidante; Transducción de señal.

enzima funcional, que após transferir elétron para o oxigênio molecular, produz o ânion superóxido. Outras fontes de formação de EROs são: xantina oxidase, uma enzima expressa na parede vascular e também circulante no plasma; mieloperoxidase, uma hemeproteína expressa em neutrófilos e monócitos que é secretada durante ativação dessas células, óxido nítrico sintase endotelial (eNOS) que, sob condições fisiológicas, produz óxido nítrico $(\mathrm{NO})$ que interage com EROs, gerando peroxinitritito $(\mathrm{ONOO} \bullet)^{2-4}$

No organismo humano, o sistema de defesa antioxidante consiste em uma série de enzimas antioxidantes além de numerosos compostos antioxidantes endógenos e de origem dietética que reagem com as EROs neutralizando-as. O sistema de defesa antioxidante enzimático inclui superóxido dismutase (SOD), glutationa peroxidase (GSH-Px) e catalase. As SOD constituem a primeira linha de defesa antioxidante e o maior sistema de defesa do organismo contra o ânion superóxido por catalisar a dismutação do ânion superóxido $\left(\bullet \mathrm{O}_{2}\right)$ em oxigênio $\left(\mathrm{O}_{2}\right)$ e peróxido de hidrogênio $\left(\mathrm{H}_{2} \mathrm{O}_{2}\right){ }^{4-6}$ Existem três isoformas de SOD, a Mn-SOD mitocondrial, a $\mathrm{Cu} / \mathrm{Zn}$-SOD citoplasmática e a SOD extracelular. $\mathrm{OH}_{2} \mathrm{O}_{2}$ formado pela dismutação do $\bullet \mathrm{O}_{2}$ pela SOD é convertido em água $\left(\mathrm{H}_{2} \mathrm{O}\right)$ e $\mathrm{O}_{2}$ pela catalase, uma enzima antioxidante localizada em peroxissomos celulares. Além da catalase, a glutationa peroxidase também remove $\mathrm{H}_{2} \mathrm{O}_{2}$ por acoplar sua redução com a oxidação da glutationa reduzida (GSH). A GSH-Px é uma enzima antioxidante que contém selênio e, além de reduzir efetivamente $\mathrm{o}_{2} \mathrm{O}_{2}$, reduz também peróxidos lipídicos a lipídios alcoólicos. Essas enzimas estão presentes no citoplasma em concentrações milimolares e também estão presentes na matriz mitocondrial. O desequilíbrio redox está envolvido na patogênese e progressão de distintas doenças, incluindo as neurodegenerativas, o câncer, as cardiovasculares, o diabetes mellitus e a hipercolesterolemia. Estas patologias apresentam como características em comum a inflamação crônica que, juntamente com o estresse oxidativo, formam um binômio importante no desenvolvimento das mesmas.?

Após exposição celular a EROs e alteração no status redox, há o desencadeamento do processo de transcrição de genes inflamatórios e os fatores de 


\section{Artigo de revisão}

transcrição regulados através de mecanismos redoxsensíveis. ${ }^{7}$ Neste sentido, a ativação do nuclear factor kappa B (NF-kB) por EROs estimulam a transcrição de um grande número de genes que codificam citocinas inflamatórias e moléculas de adesão. ${ }^{8,9}$ Assim, quando o NF-kB é ativado, várias citocinas pró-inflamatórias, tais como o fator de necrose tumoral alfa (TNF-a), interleucinas (IL) 1, 2, 6 e 12 são produzidos em excesso. ${ }^{10}$ Além disso, as próprias citocinas podem levar à formação de EROs, que estabelece um ciclo vicioso entre o estresse oxidativo e a geração de citocinas pró-inflamatórias. Na ausência de estímulo, o NF-kB se encontra no citoplasma ligado a sua proteína repressora IkB ( $\mathrm{IkBa}$, IkBb, IkBg, IkB 3, e Bcl3). ${ }^{11,12}$ Desta forma, a investigação acerca de estratégias que promovem a quebra deste ciclo vicioso são fundamentais. Inserido neste contexto, o nuclear factor-erythroid 2-related factor-2 (Nrf2) é um fator de regulação positivo para o elemento de resposta antioxidante (ARE), regulando a expressão de enzimas antioxidantes. $^{13}$

Ambos os fatores de transcrição e suas possíveis vias de sinalização celular têm sidos descritos como alvos para o desenvolvimento de medicamentos e pesquisas científicas, principalmente, após a conclusão do Projeto Genoma Humanas no ano de 2003. O conhecimento das características genéticas humanas trouxe uma nova perspectiva para redução do risco de doenças crônicas não transmissíveis (DCNT). Considerando-se que o desenvolvimento de doenças como as cardiovasculares, diabetes e obesidade envolve componentes tanto genéticos quanto ambientais, grande ênfase tem sido dada, atualmente, à elucidação da interação entre genes e fatores ambientais. ${ }^{14,15}$

Um dos principais fatores ambientais a que o ser humano é submetido diariamente é a alimentação. $\mathrm{O}$ tipo e a qualidade dos alimentos ingeridos são de extrema importância, não apenas pelo seu valor nutricional, mas também pela capacidade de seus nutrientes e compostos bioativos em interagirem com ogenoma. Assim, a Nutrigenômica representa uma vertente de estudos no âmbito nutricional, que tem como foco estudar a interação gene-nutriente. Essa ocorre de duas formas complementares: nutrientes e compostos bioativos dos alimentos (CBAs) modulam o funcionamento do genoma e, da mesma forma, características do genoma influenciam a resposta à alimentação, necessidade de nutrientes e risco para DCNT. ${ }^{16,17}$

Diferentes alimentos contêm nutrientes e compostos bioativos com ação anti-inflamatória, sendo parte desse efeito devido à modulação da via de sinalização de fatores de transcrição envolvidos em vias pro e anti-inflamatórias. Junto a isso, a compreensão de como antioxidantes atuam em vias moleculares em respostas ao estresse oxidativo é extremamente importante para permitir o desenvolvimento de novas alternativas terapêuticas de tratamento e/ou prevenção. A base para terapias antioxidantes reside no reequilíbrio no ambiente redox e no reestabelecimento da alteração existente em vias de sinalização celular. Assim, o papel do estresse oxidativo na regulação da transcrição gênica está se tornando cada vez mais importante. Isso não só fornece uma visão ampliada sobre o papel fisiológico do estresse oxidativo, mas também apresenta sistemas regulatórios que são fundamentais na manutenção da homeostasia.

\section{VIA NRF2/KEAP1}

O Nrf2 é um fator de transcrição nuclear que se encontra inativo no citoplasma ligado à proteína Kelch-like ECH-associated protein 1 (Keap1) a qual impede sua translocação para o núcleo. Este fator é ativado por alterações do estado redox da célula, tais como o aumento das concentrações de EROs, subprodutos de danos oxidativos e reduzida capacidade antioxidante, e compostos bioativos vem sendo potenciais promotores de sua resposta transcricional no elemento de resposta antioxidante (ARE), perdendo, assim, a ligação com a Keap1 (Figura 1). ${ }^{18} \mathrm{O}$ ARE é um componente vital das regiões promotoras dos genes que codificam enzimas detoxificantes e antioxidantes. Assim, o Nrf2 atua quando liberado de sua proteína citosólica repressora (Keap1). Depois de ter sido translocado para o núcleo, o Nrf2 ativa os genes que codificam enzimas de fase II, detoxificantes e enzimas antioxidantes, que incluem: superóxido dismutase (SOD), catalase (CAT), NADPH quinona oxidorredutase 1 (NQO1), heme oxigenase-1 (HO-1), glutationa peroxidase (GPx), e tioredoxina (Trx), dentre outras, as quais desempenham papéis fundamentais na defesa celular, atenuando os efeitos adversos de EROs.?

Em condições basais, um dos mecanismos propostos de regulação da via é que o Nrf2 seja constantemente ubiquitinizado, através da Keap1 no citoplasma e degradado no proteossomo. ${ }^{19}$ Quando as células são expostas à tensão oxidativa ou eletrofílica, os resíduos de cisteína da Keap1 são modificados e perdem a sua capacidade de ubiquitinizar o Nrf2. Este, então, acumula- se no interior da célula e migra ao núcleo para induzir a expressão dos seus genes-alvo. O sistema de 
regulação tem sido referido como o sistema Keap1Nrf2, que desempenha um papel central na proteção de células contra o estresse. ${ }^{20}$

Atualmente, o sistema Nrf2/Keap1 tem sido reconhecido como um dos principais mecanismos de defesa celular contra o estresse oxidativo. Muitos genes regulados pelo Nrf2/ARE desempenham papéis importantes envolvidos na modulação da via de sinalização redox e na inflamação crônica. É provável que a função citoprotetora de genes alvo de Nrf2 possa, cooperativamente, regular a resposta imune inata e também reprimir a indução de genes pró-inflamatórios. ${ }^{711}$ Como um exemplo, a tioredoxina reduziu EROs, tais como peróxido de hidrogênio $\left(\mathrm{H}_{2} \mathrm{O}_{2}\right)$, protegendo as células de TNF-a e da lesão provocada pelo processo de isquemia-reperfusão. ${ }^{21}$ Além disso, a superexpressão de NQO1 em células endoteliais inibiu a expressão induzida por TNF-a de VCAM-1, conferindo proteção contra processos inflamatórios na parede vascular. ${ }^{22}$

Outra importância do Nrf2 tem sido associada à melhora do perfil inflamatório por sua capacidade de antagonizar o NF-kB. Mao e colaboradores, ${ }^{23}$ utilizando um modelo Nrf2 (-/-), observou um aumento da ativação e na expressão de NF-kB, TNF-a, e MMP-9 após a lesão da medula espinhal. Em outro estudo, ${ }^{24} \mathrm{o}$ rato knockout (KO) para Nrf2 apresentou maior ativação de NF-kB, citocinas inflamatórias TNF-a, IL-1b, IL-6, e ICAM-1 após lesão cerebral traumática.
Estudos demonstram que compostos bioativos presentes em alimentos podem modular a via Nrf2/ Keap1, atuando, assim, indiretamente no estresse oxidativo e na expressão do fator de transcrição NFkB, atuante na resposta inflamatória. Estes achados são fundamentais não só para mostrar os efeitos benéficos dos fitoquímicos presentes naturalmente em alimentos, como também a importância desta via de sinalização em patologias que estão cada vez mais prevalentes, como as doenças cardiovasculares, as neurodegenerativas, o diabetes, a hipertensão, entre outras. Com isso, uma vez demonstrada a associação destes, garante-se a contribuição para o desenvolvimento de novas terapias nutricionais.

Os polifenóis se enquadram no grupo dos compostos bioativos encontrados na literatura, mas existem outros CBAs, como: a alicina, a quercetina, o licopeno, entre outros. ${ }^{6}$ Os polifenóis estão presentes em alimentos de origem vegetal, com cerca de 8.000 compostos já identificados, que representam um grande grupo de metabolitos secundários, consistindo em um ou mais anéis aromáticos com graus variáveis de hidroxilação, metilação e glicosilação, contribuindo com a cor, adstringência e amargor do fruto. As principais categorias de compostos fenólicos são: os ácidos fenólicos, flavonóides, taninos e estilbenos, porém estas ainda sofrem subdivisões. ${ }^{25}$ Esses compostos agem como antioxidantes, não somente pela sua habilidade em doar

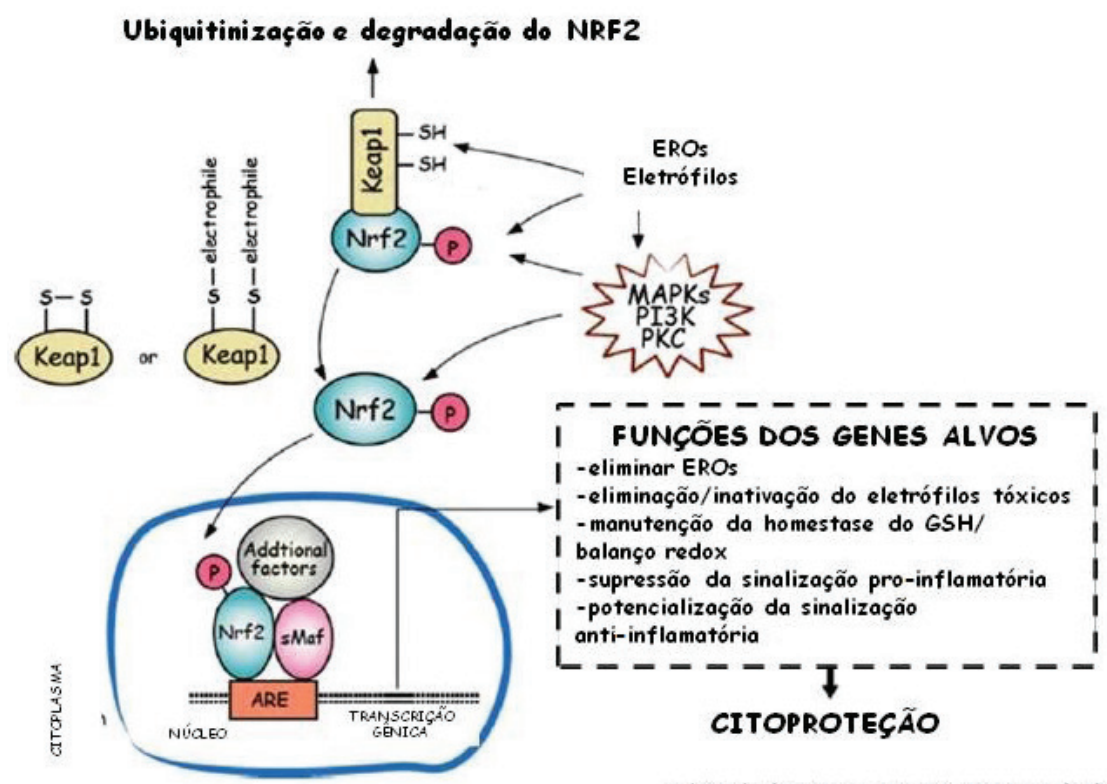

Figura 1. . Mecanismo proposto responsável pela ativação da via de sinalização do Nrf2/ARE 


\section{Artigo de revisão}

elétrons, mas também de forma indireta, em virtude de ser um potencial modulador de vias de sinalização antioxidante e anti-inflamatória, como as supracitadas. A seguir, estão descritos alguns compostos bioativos presentes em alimentos que potencialmente regulam a expressão de genes alvos no processo de estresse oxidativo e inflamação.

\section{Curcumina}

A curcumina é um composto presente naturalmente no açafrão (Curcuma longa), com uma longa história de uso na tradicional alimentação indiana e na fitoterapia. Existem várias propriedades farmacológicas e biológicas, descritas na literatura, tais como antioxidante, anti-inflamatória, anticancerígeno, antimicrobianos, antivirais e antifúngicos. ${ }^{26}$

Utilizando modelo in vitro (células H9C2) e in vivo (camundongos C57BL/6) Zeng e colaboradores observaram que, após estresse induzido por palmitato e dieta hiperlipídica, o tratamento com curcumina melhorou significativamente a hipertrofia cardíaca, apoptose e fibrose associada à lesão induzida pelos agentes agressores. ${ }^{27}$

A proteção cardíaca conferida por este CBA foi mediada, provavelmente, por ativação de Nrf2 e inibidores de ação do NFkb. Fato atribuído ao aumento da expressão proteica e do RNA mensageiro (RNAm) de Nrf2, seguido do aumento de enzimas antioxidantes (HO-1, GCLC, NQO1) e diminuição da forma fosforilada de NFkB (NFkBp65) in vitro; além de maior expressão de IkB-a (proteína repressora do NFkB) no tecido cardíaco do modelo in vivo.

O estudo de Boonla e colaborares objetivou investigar os efeitos da curcumina em modelo in vivo de hipertensão renovascular. ${ }^{28}$ As principais conclusões foram de que o tratamento com a curcumina: atenuou o aumento da pressão arterial sistólica, a disfunção endotelial e melhorou a remodelamento vascular. Estes efeitos benéficos foram observados graças a melhoria do estado hemodinâmico, atenuação de alteração estrutural vascular (diminuição na deposição de colágeno e elastina), diminuição na expressão da enzima conversora de angiotensina (ECA) e dos níveis de MMPs 2 e 9, atenuação do estresse oxidativo por redução na formação de superóxido, associada, por sua vez, a menor expressão da subunidade p47phox do complexo enzimático NADPH oxidase; além de diminuir biomarcadores de estresse (malondialdeído e proteínas carboniladas). Também foi observado aumento na biodisponibilidade de óxido nítrico através de uma maior expressão de óxido nítrico sintase endotelial (eNOS), contribuindo para maior relaxamento vascular.

Em células do adenocarcinoma mamário (MCF-7), Cheng e colaboradores demonstraram que a proliferação celular desta linhagem depende de Fen1, uma das enzimas envolvidas na replicação e reparação do DNA, e que diminui após exposição ao seu RNA de interferência (siRNA). ${ }^{29}$ Além disso, a curcumina atenuou significativamente a atividade do promotor Fen1 e expressão da proteína de forma dose dependente. Portanto, por diminuir a expressão de Fen1, sugere-se um novo mecanismo pelo qual este composto inibe a proliferação de células cancerígenas mamárias. Os resultados ainda mostram que a baixa regulação de Fen1 induzida pela curcumina em células MCF-7 foi mediada por Nrf2. O Nrf2 também desempenha um papel importante no desenvolvimento e progressão de tumores, uma vez que muitos genes envolvidos na carcinogênese foram confirmados por ser controlados direta ou indiretamente por Nrf2.

\section{Resveratrol}

O resveratrol (3, 5, 4'-trihidroxiestilbeno) é um composto derivado da classe dos polifenóis encontrados em várias plantas, incluindo as uvas, no vinho tinto, no amendoim e nos pinheiros. ${ }^{6}$

Este composto bioativo conferiu proteção às células epiteliais da córnea humana (HLEB-3) contra danos oxidativos induzidos por peróxido de hidrogênio $\left(\mathrm{H}_{2} \mathrm{O}_{2}\right)$, e o mecanismo de proteção ocorreu pela indução de três enzimas antioxidantes: a superóxido dismutase, heme-oxigenase 1 e a catalase (SOD-1, HO-1e CAT). Logo, surge assim um possível agente terapêutico contra o desenvolvimento da catarata. ${ }^{30}$

O tratamento profilático com resveratrol atenuou significativamente a lesão miocárdica induzida por endotoxina em camundongos, associada à supressão da produção de citocinas pró-inflamatórias e aumento na ativação do Nrf2 no coração. Em uma cultura primária de cardiomiócitos humanos, este CBA ativou o Nrf2 e inibiu a geração de EROs induzida por lipopolissacarídeo (LPS), sendo uma forma eficaz de proteção celular contra a apoptose condicionada por LPS. O knockout de Nrf2 anulou a proteção mediada pelo resveratrol contra a morte celular anteriormente citada, o que sugere um papel essencial deste CBA, junto com o Nrf2 na cardioproteção. ${ }^{31}$

Palsamy e Subramanian, em modelo animal (ratos Wistar machos) de diabetes induzida por estreptozocina (intraperitoneal $50 \mathrm{mg} / \mathrm{kg}$ ), administraram resvera- 
trol via oral $(5 \mathrm{mg} / \mathrm{kg}$ ) e observaram uma diminuição significativa do cleareance da creatinina, dos níveis de adiponectina plasmática, do peptídeo $\mathrm{C}$, do ânion superóxido, do radical hidroxila, do óxido nítrico, e de TNF-a, IL-1 $\beta$, IL-6 e NF-kBp65 renal, em comparação com ratos diabéticos sem tratamento. ${ }^{32}$

Além disso, o tratamento com resveratrol revelou melhora importante da atividade de enzimas antioxidantes (SOD, CAT, GPx) com uma diminuição significativa em peróxidos lipídicos, hidroperóxidos e proteínas carboniladas em rins diabéticos. Do mesmo modo, o CBA normalizou a expressão renal de Nrf2/Keap1 no grupo diabético. Observações histológicas evidenciaram a proteção renal contra o dano oxidativo mediado por hiperglicemia. Estes resultados demonstram a natureza renoprotetora desse composto, atenuando marcadores de estresse oxidativo nos tecidos renais de ratos diabéticos.

Evidências recentes apontam o Nrf2 como alvo do resveratrol.Em cultura de células endoteliais da artéria coronariana, este composto promoveu, de maneira dose-dependente, aumento na atividade transcricional de Nrf2 e, consequentemente, na expressão de enzimas antioxidantes (NQO1, HO1). No modelo in vivo, após receberem dieta hiperlipídica, os camundongos knockouts para Nrf2 tiveram o efeito protetor do resveratrol diminuído. Estes resultados ratificam que este CBA tanto in vivo quanto in vitro conferem efeito protetor ao endotélio mediado pela ativação de Nrf2.33

\section{Sulforafano}

Sulforafano é um composto organossulfurado encontrado em crucíferas (repolho, couve-de-bruxelas, brócolis, entre outros). O consumo desses alimentos é implicado na diminuição do risco de doenças crônicas não transmissíveis, dentre elas o diabetes e a doença cardiovascular. ${ }^{34}$

No estudo de Zheng e colaboradores, foi observado que camundongos que inalaram arsênio (AS) e receberam injeção intraperitoneal de $10 \mathrm{mg} / \mathrm{kg} / \mathrm{dia}$ de sulforafano (SF) tiveram maior expressão de Nrf2 e seus genes alvos (NQO1, HO- $1, \gamma$-GCS), resultado ainda mais expressivo em animais que receberam AS + SF. ${ }^{35}$ Em animais knockouts para Nrf2, não foi detectado Nrf2 e seus genes alvo tiveram baixa expressão. Além disso, alterações patológicas, como: maior espessura da camada de células epiteliais alveolares, bem como do septo alveolar e aumento de citocinas pró-inflamatórias (TNF-a, IL-6, IL-13, IL-4, TGF- $\beta$, MCP-1) foram observadas nos grupos não tratados. Houve, ainda, maior ativação de NFkBp65, e o SF preveniu a fosforilação da p65 induzida por AS, com exceção do grupo knockout. No grupo Nrf2-KO, o composto não foi capaz de reverter o quadro patológico supracitado, sugerindo então que o SF atua de maneira Nrf2-dependente. Assim, percebe-se que a exposição ao AS resultou em alterações patológicas e intracelulares importantes em tecido pulmonar, sendo todos os efeitos atenuados por SF, em uma atuação dependente do Nrf2.

Bai e colaboradores visaram avaliar se SF pode prevenir o desenvolvimento de cardiomiopatia diabética. Após indução do diabetes (DM) tipo I via injeção intraperitoneal de estreptozotocina em camundongos oSF foi administrado por três meses $(0,5 \mathrm{mg} / \mathrm{kg} / \mathrm{dia}$, durante 5 dias na semana) e após 6 meses algumas análises foram refeitas. ${ }^{34}$

Os principais resultados observados foram diminuição da pressão arterial, prevenção das disfunções cardíacas e reversão da indução a hipertrofia cardíaca nos grupos diabéticos, após administração do CBA. Junto a isso, a expressão de Nrf2 em tecido cardíaco no grupo DM foi maior, porém diminuiu ao final dos seis meses de diabetes. Já no grupo DM/SF, em comparação ao grupo DM, houve aumento da expressão ao final dos seis meses.

Em cultura de células expostas a ambiente hiperglicêmico, houve aumento de efeito fibrótico, quase completamente revertido por tratamento com SF, e ainda aumento da expressão do Nrf2. Quando se inibiu a expressão deste fator de transcrição, houve perda da prevenção dos efeitos do composto em meio hiperglicêmico.

Assim, os resultados demonstraram que SF pode proteger contra danos ocasionados por diabetes, podendo prevenir o desenvolvimento da cardiomiopatia diabética, bem como promover um aumento na expressão de Nrf2 em tecido cardíaco.

Para avaliar a atividade deste CBA como um ativador de Nrf2 na esteatose induzida por etanol, Zhou e colaboradores utilizaram um modelo knockin com concentrações elevadas de citocromo P450 2E1 (CYP2E1), justificando ser de mais fácil visualização não só os efeitos do etanol no estresse oxidativo e injúria no fígado, como também os do suforafano e um modelo in vitro que adicionou sulforafano em células HepG2 E47 que expressam CYP2E1. ${ }^{36}$

O tratamento com SF ativou Nrf2 e aumentou os níveis de HO-1 e, como consequência, diminuiu o dano oxidativo por atenuar a peroxidação lipídica e por aumento nos níveis de GSH depois do tratamento 


\section{Artigo de revisão}

com etanol. Além disso, reduziu níveis hepáticos de triglicerídeos e colesterol que aumentaram por indução do etanol. Resultados similares foram observados com a adição do SA na linhagem celular, que também apresentou maior expressão de Nrf2 e diminuição no acúmulo de lipídeos nas células tratadas com etanol.

\section{Epigalocatequina-galato (EGCG)}

A EGCG é um composto bioativo encontrado principalmente no chá verde que apresenta esta catequina em maior concentração. Muitos estudos atribuem a este CBA as propriedades biológicas conferidas ao chá verde como seu potencial antioxidante e anti-inflamatório. ${ }^{37}$

Em um estudo que avaliou o efeito citoprotetor de EGCG contra a toxicidade induzida por deoxinivelanol (DON), em linhagem celular de adenocarcinoma intestinal (HT-29) ${ }^{38}$ foi demonstrada melhora do desequilíbrio oxidativo induzido por DON, uma micotoxina encontrada em alimentos, graças a: diminuição na geração de EROs, maior integridade da membrana quando comparado ao controle, avaliada pelo ensaio de lactato desidrogenase (LDH), menor produção de nitrito e proteínas carboniladas; além da manutenção dos níveis de glutationa (GSH) próximos ao do grupo controle. O grupo que recebeu este composto apresentou menor atividade enzimática antioxidante (SOD, CAT, GPx, GST) quando comparados ao grupo que recebeu somente DON, sugerindo assim um efeito benéfico direto de EGCG.

Ressalta-se ainda que marcadores inflamatórios, como NFkBp65 e COX 2, foram menos expressos no grupo tratado, levando em consideração que uma vez diminuída a geração de EROs a menor expressão de NFkBp65 é esperado, haja visto que as espécies reativas são fundamentais para a via de sinalização inflamatória mediada por este fator de transcrição.

Han e colaboradores tiveram como principal hipótese a proteção de EGCG contra injúria induzida por isquemia e a melhora do dano oxidativo através da ativação da via Nrf2/ARE. ${ }^{39}$ Assim em modelo in vivo (ratos Sprague-Dawley) ocluíram a artéria cerebral média e trataram os animais com 40mg/kg de EGCG (via intraperitoneal). Observaram um efeito protetor do CBA na injúria cerebral induzida pelo processo de isquemia/reperfusão, devido a: melhora do quadro neurológico, diminuição da área de infarto cerebral e atenuação da injúria neuronal no córtex isquêmico. $\mathrm{O}$ grupo tratado manteve os níveis de GSH próximos ao do grupo controle. Além disso, houve maior expressão de Nrf2 e de enzimas antioxidantes no grupo com
EGCG, garantindo uma melhora nostatus antioxidante em tecido cerebral e sugerindo que esta seja mediada pela ativação da via de sinalização do Nrf2.

Já Thangapandiyan e Miltonprabu abordam o EGCG como um protetor contra a nefrotoxicidade induzida por fluoreto (Fl), através de modelo animal (ratos Wistar). ${ }^{40}$ Demonstraram que o tratamento com EGCG diminuiu as concentrações séricas de ureia, ácido úrico e creatinina bem como o clearance de creatinina, aumentando a concentração urinária destes marcadores nefrotóxicos. Houve diminuição, em tecido renal, de citocinas pró-inflamatórias (TNF-a, NO, IL-6 e NFkBp65). Além disso, os produtos de peroxidação lipídica encontraram-se diminuídos no grupo Fl tratado com EGCG quando comparado ao não tratado. Entretanto, neste caso a pré-administração deste CBA no grupo que não recebeu Fl diminuiu significativamente estes marcadores de estresse oxidativo quando comparados ao controle, sugerindo assim um efeito benéfico do CBA mesmo sem um indutor de estresse. Observaram ainda aumento na concentração de enzimas antioxidantes (SOD, CAT, GPx, GST e glutationa redutase (GR) no grupo tratado. Junto a isso a EGCG foi capaz de normalizar, quase que totalmente, a expressão tanto a nível de RNAm, quanto de proteínas de Nrf2, HO-1, ү-GCS e $\mu$-GST, com diminuição da Keap1. Houve diminuição da expressão de Bax (B-cell associated X protein) e aumento na de Bcl-2 (B-cell lymphoma 2), sugerindo uma contra regulação ao mecanismo de apoptose, visto que a Bcl-2 esta relacionada à inibição deste mecanismo de morte celular; além de outros marcadores de apoptose como citocromo C e as caspases 9 e 3 . Assim, há uma proteção renal deste CBA contra a injúria induzia pelo íon de flúor e que a ativação da via Nrf2/Keap1 pelo EGCG pode ser responsável pela atenuação do estresse oxidativo mediado pelo fluoreto com consequente inflamação renal e apoptose. Isso graças a possível interação entre o CBA e os resíduos de cisteína presentes na Keap 1 que permitem a translocação nuclear do Nrf2.

Portanto, podemos ressaltar a partir dos dados descritos na literatura que os CBAs atuam beneficamente no reestabelecimento do desequilíbrio redox, atuando concomitantemente ou não, na modulação de vias de sinalização moleculares antioxidantes e inflamatória, apresentando potencial uso terapêutico em comorbidades que estão cada vez mais prevalentes em países em desenvolvimento. Todavia mais estudos são necessários para se esclarecer doses toxicológicas e ainda elucidar o impacto destes compostos em seres humanos. 
Elaine dos R. Soares e cols. • Compostos bioativos em alimentos, estresse oxidativo e inflamação: uma visão molecular da nutrição

\section{Referências}

1. Cunningham FG, Leveno KJ, Blomm SL, et al. Tireóide e outros distúrbios endócrinos. In: Cunningham FG et al. Obstetrícia de Williams. 23 ed. Porto Alegre: Artmed-McGraw-Hill, 2012, p.1126-44.

2. Mestman JH. Thyroid and Parathyroid Diseases in Pregnancy. In: Gabbe SG; Niebyl JR; Simpson JL; Landon MB; Galan HL; Jauniaux ERM et al. Obstetrics normal and problem pregnancies. 6th ed. Saunders Elsevier: Philadelphia, 2012, p.922-53.

3. Fitzpatrick DL, Russell MA. Diagnosis and management of thyroid disease in pregnancy. Obstet Gynecol Clin North Am. 2010;37(2):173-93. doi: 10.1016/j.ogc.2010.02.007.

4. Stagnaro-Green A, Abalovich M, Alexander E, et al.Guidelines of the American Thyroid Association for the diagnosis and management of thyroid disease during pregnancy and postpartum. Thyroid. 2011;21(10):1081-125. doi: 10.1089/ thy.2011.0087. Epub $2011 \mathrm{Jul} 25$.

5. Casey BM, Dashe JS, Spong CY, et al. Perinatal significance of isolated maternal hypothyroxinemia identified in the first half of pregnancy. Obstet Gynecol. 2007; 109(5):1129-35.

6. Schneuer FJ, Nassar N, Tasevski V, et al. Association and predictive accuracy of high TSH serum levels in first trimester and adverse pregnancy outcomes. J Clin Endocrinol Metab. 2012; 97(9):3115-22. doi: 10.1210/jc.2012-1193. Epub 2012 Jun 20.

7. De Groot L, Abalovich M, Alexander EK, et al. Management of thyroid dysfunction during pregnancy and postpartum: an Endocrine Society clinical practice guideline. J Clin Endocrinol Metab. 2012; 97(8):2543-65. doi: 10.1210/jc.2011-2803.

8. Negro R, Schwartz A, Gismondi R, et al. Increased pregnancy loss rate in thyroid antibody negative women with TSH levels between 2.5 and 5.0 in the first trimester of pregnancy. J Clin Endocrinol Metab. 2010; 95(9):E44-8. doi: 10.1210/jc.20100340. Epub 2010 Jun 9.

9. Seror J, Amand G, Guibourdenche J, et al. Anti-TPO antibodies diffusion through the placental barrier during pregnancy. PLoS One. 2014; 31:9(1):e84647. doi: 10.1371/journal.pone.0084647.

10. Nor Azlin MI, Bakin YD, Mustafa N, et al. Thyroid autoantibodies and associated complications during pregnancy. J Obstet Gynaecol. 2010; 30(7):675-8. doi: 10.3109/01443615.2010.503908.

11. Hirsch D, Levy S, Nadler V, et al. Pregnancy outcomes in women with severe hypothyroidism. Eur J Endocrinol. 2013;169(3):313-20. doi: 10.1530/EJE-13-0228.

12. Alexander EK, Marqusee E, Lawrence J, et al. Timing and magnitude of increases in levothyroxine requirements during pregnancy in women with hypothyroidism. N Engl J Med. 2004;351(3):241-9.

13. Vadiveloo T, Mires GJ, Donnan PT, et al. Thyroid testing in pregnant women with thyroid dysfunction in Tayside, Scotland: the thyroid epidemiology, audit and research study (TEARS). Clin Endocrinol (Oxf). 2013; 78(3):466-71. doi: 10.1111/j.13652265.2012.04426.x.

14. Loh JA, Wartofsky L, Jonklaas J, et al. The magnitude of increased levothyroxine requirements in hypothyroid pregnant women depends upon the etiology of the hypothyroidism. Thyroid 2009; 19(3):269-75. doi: 10.1089/thy.2008.0413.

15. Kaplan MM. Management of thyroxine therapy during pregnancy. Endocr Pract. 1996; 2(4):281-6.

16. Chávez A, Chávez MM. Nutrigenomics in public health nutrition: short-termperspectives. European Journal of Clinical Nutrition. 2003;57(Supl 1):S97-100.
17. Müller M, Kersten S. Nutrigenomics: goals and strategies. Nature Reviews. 2003 Abr;4:315-22.

18. Wilmes A, Crean D, Aydin S, et al. Identification and dissection of the Nrf2 mediated oxidative stress pathway in human renal proximal tubule toxicity. Toxicol In Vitro. 2011 Abr;25(3):613-22.

19. Mitsuishi Y, Motohashi H, Yamamoto M. The Keap1-Nrf2 system in cancers: stress response and anabolic metabolism. Frontiers in Oncology.2012 Dez 26; 2:200. Pubmed: PMID: 23272301.

20. Uruno A, Yagishita Y, Yamamoto M. The Keap1-Nrf2 system and diabetes mellitus. Archives of Biochemistry and Biophysics. 2014;566:76-84.

21. Mitsui A, Hirakawa T, Yodoi J. Reactive oxygen-reducing and protein refolding activities of adult $\mathrm{T}$ cell leukemia-derived factor/human thioredoxin, Biochem. Biophys. Res. Commun. 1992;186:1220-26.

22. Chen XL, Varner SE, Rao AS, et al. Laminar flow induction of antioxidant response element-mediated genes in endothelial cells: a novel antiinflammatory mechanism. J. Biol. Chem. 2003;278:703-11.

23. Mao L, Wang H, Qiao L, et al. Disruption of Nrf2 enhances the upregulation of nuclear factor-kappaB activity, tumor necrosis factor-a, and matrix metalloproteinase- 9 after spinal cord injury in mice. Mediators Inflamm.2010;1-10. Pubmed: PMID: 20862369.

24. Jin W, Wang H, Yan W, et al. Disruption of Nrf2 enhances upregulation of nuclear factor-kappaB activity, proinflammatory cytokines, and intercellular adhesion molecule- 1 in the brain after traumatic brain injury. Mediators Inflamm. 2008:1-7. Pubmed: PMID:19190763

25. Manganaris GA, Goulas V, Vicente AR, et al. Berry antioxidants: small fruits providing large benefits. J Sci Food Agric. 2014 Mar 30;94(5):825-33.

26. Prasad S, Gupta SC, Tyagi AK, et al. Curcumin, a component of golden spice: from bedside to bench and back. Biotechnol Adv 2014;32:1053-64.

27. Zeng $C$, Zhong $P$, Zhao $Y$, et al. Curcumin protects hearts from FFA-induced injury by activating Nrf2 and inactivating NF- B both in vitro and in vivo. Journal of Molecular and Cellular Cardiology. 2014;79:1-12.

28. Boonla O, Kukongviriyapan U, Pakdeechote P, et al. Curcumin improves endothelial dysfunction and vascular remodeling in $2 \mathrm{~K}-1 \mathrm{C}$ hypertensive rats by raising nitric oxide availability and reducing oxidative stress. Nitric Oxide.2014;42:44-53.

29. Chen B, Zhang Y, Wang Y, et al. Curcumin inhibits proliferation of breast cancer cells through Nrf2-mediated down-regulation of Fen1 expression. Journal of Steroid Biochemistry and Molecular Biology. 2014;143:11-18.

30. Zheng Y, Liu Y, Ge J. Resveratrol protects human lens epithelial cells against $\mathrm{H} 2 \mathrm{O} 2$ - induced oxidative stress by increasing catalase, SOD-1, and $\mathrm{HO}-1$ expression. Molecular Vision.2010;16:1467-74.

31. Hao E, Lang F, Chen Y, et al. (2013) Resveratrol Alleviates Endotoxin-Induced Myocardial Toxicity via the Nrf2 Transcription Factor. PLoS ONE.2013 Jul 02. 8(7): e69452. Pubmed: PMID: 23894482

32. Palsamy P, Subramanian S. Resveratrol protects diabetic kidney by attenuating hyperglycemia-mediated oxidative stress and renal inflammatory cytokines via Nrf2-Keap1 signaling. Biochimica et Biophysica Acta. 2011;1812:719-31.

33. Ungvari Z, Bagi Z, Feher A, et al. Resveratrol confers endothelial protection via activation of the antioxidant transcription 


\section{Artigo de revisão}

factor Nrf2. Am J Physiol Heart Circ Physiol. 2010; 299:H18-24.

34. Bai Y, Cui W, Xin Y, et al. Prevention by sulforaphane of diabetic cardiomyopathy is associated with up-regulation of Nrf2 expression and transcription activation. Journal of Molecular and Cellular Cardiology. 2013;57:82-95.

35. Zheng Y, Tao S, Lian F, et al. Sulforaphane prevents pulmonary damage in response to inhaled arsenic by activating the Nrf2-defense response. Toxicology and Applied Pharmacology. 2012;265:292-299.

36. Zhou R, Linb J, Wua D. Sulforaphane induces Nrf2 and protects against CYP2E1-dependent binge alcohol-induced liver steatosis. Biochimica et Biophysica Acta. 2014;1840:209-18.

37. Annaba F, Kumar P, Dudeja AK, et al. Green tea catechin EGCG inhibits ileal apical sodium bile acid transporter
ASBT. Am J Physiol Gastrointest Liver Physiol. 2010 Mar; 298(3):G467-73. Pubmed: PMCID: PMC2838517

38. Kalaiselvi P, Rajashree K, Priya LB, et al. Cytoprotective effect of epigallocatechin-3-gallate against deoxynivalenol-induced toxicity through anti-oxidative and anti-inflammatory mechanisms in HT-29 cells. Food and Chemical Toxicology. 2013;56:110-18.

39. Han J, Wang M, Jing X, et al. (2)-Epigallocatechin Gallate Protects Against Cerebral Ischemia- Induced Oxidative Stress via Nrf2/ARE Signaling. Neurochem Res. 2014;39:1292-99.

40. Thangapandiyan S, Miltonprabu S. Epigallocatechin gallate supplementation protects against renal injury induced by fluoride intoxication in rats: Role of $\mathrm{Nrf} 2 / \mathrm{HO}-1$ signaling. Toxicology Reports 1. 2014;12-30. 